\title{
AN ANALYSIS OF ZMP CONTROL PROBLEM \\ OF HUMANOID ROBOT WITH COMPLIANCES IN SOLE OF THE FOOT
}

\author{
Napoleon Nazir* Hiroki Izu ${ }^{* *}$ Shigeki Nakaura* \\ Mitsuji Sampei* \\ * Department of Mechanical \& Control Engineering, \\ Tokyo Institute of Technology \\ ** Toyota Motor Corporation \\ Email: leo@sc.ctrl.titech.ac.jp
}

\begin{abstract}
This paper describes an analysis of balance control of humanoid robot based on ZMP feedback control. The purpose of this paper is to make clear understanding of ZMP control problem which is composed by inverted pendulum control and ZMP compensation control. This paper shows that this problem can be formulated as high gain output feedback and the existence of zero dynamics in closed loop system. This paper also shows stability condition of zero dynamics, and then stability of zero dynamics and closed-loop systems are analyzed using root locus. From the root locus analysis, the guidelines on finding state and output feedback gain which achieve good performance of control system, are shown. Copyright (C)2005 IFAC
\end{abstract}

Keywords: Humanoid robot, ZMP feedback control, Zero dynamics, Control system analysis, Root locus

\section{INTRODUCTION}

Zero Moment Point(ZMP) principle proposed by Vukobratovic to consider stability of leg systems (Vukobratovic et al. (1975)), is defined as the point in the ground where the resultant moments to system are zero. Even if the stability based on ZMP only describes contact condition between foot and the ground, ZMP based controller is mainly used in humanoid robot communities because it is known to work well experimentally.

In most of cases, the trajectories of humanoid robot are designed offline, and then controller is designed to track these trajectories. However even if stable trajectories are used, the existence of impulse disturbances on foot's sole can make robot to tumble. In many cases, stability of humanoid robot is also maintained by ZMP based controller, and one of methods which is known to work well is ZMP controller composed by inverted pendulum and ZMP compensation control (Nagasaka et al. (1999)). Inverted pendulum control is used to generate desired ZMP so that balance can be maintained while ZMP compensation control is used to track desired ZMP.
Balance can be maintained using ZMP based controller, however the meaning of ZMP controller is not clearly understand. Thus, controller parameters need to be adjusted frequently and experimentally. And because there is also feedthrough term from postural acceleration input $(\ddot{\theta})$ to $\mathrm{ZMP}(p)$, balance stability of closed loop system is difficult to analyze.

From the facts described above, this paper reformulates balance stabilization based on ZMP controller composed by inverted pendulum and ZMP compensation control, and proposes a guideline to decide controller parameters in designing ZMP controller in real application. Stability analysis of closed loop system is also described using root locus method.

This paper considers humanoid robot with compliances in sole of foot, and these compliances are used to reduce the impact effects in recent humanoid robot platform. By adopting compliances to the model, the analysis of balance stability can be conducted easily because there is no feedthrough term appear from postural acceleration input $(\ddot{\theta})$ to ZMP $(p)$. 




Fig. 1. Inverted pendulum model

\section{INVERTED PENDULUM CONTROL AND ZMP COMPENSATION CONTROL}

This paper considers balance control based on ZMP principle composed by inverted pendulum and ZMP compensation control, and theoretically similar to the one conducted by Nagasaka (Nagasaka et al. (1999)) and HONDA P3 robot (Hirai et al. (1998)). ZMP controller is mostly design using 1 link inverted pendulum model as shown in Fig.1 which describes dynamics of center of mass (COM). This section describes a brief overview of controller design based on ZMP.

Humanoid robot can move in $\theta$ direction, and assume that total mass of humanoid robot is $M$ and concentrated at position $l$ from ankle. Equation of motion can be described as,

$$
M l^{2} \ddot{\theta}-M g l \sin \theta=\tau,
$$

where, $g$ and $\tau$ are gravitational acceleration and equivalent torque to move COM respectively. Since moment inertia of inverted pendulum is always positive, then transformation from input $\tau$ to postural acceleration $\ddot{\theta}$ always exist, so between $\tau$ and $\ddot{\theta}$ can be described as one-to-one mapping. Thus, besides considering torque $\tau$ as the input to the system, it is equivalent to consider postural acceleration $\ddot{\theta}$ as input of the system. In the following formulation, postural acceleration is used as input to the system.

In humanoid robot model described above, $p$ is the position of ground reaction force which describes ZMP. Linearizing equation of motion, ZMP $(p)$ can be expressed as,

$$
p=l \theta-\frac{l^{2}}{g} \ddot{\theta} .
$$

And equivalent torque $(\tau)$ and ZMP $(p)$ have the following relation,

$$
p=-\frac{1}{M g} \tau .
$$

\subsection{Inverted pendulum control}

Inverted pendulum control is used to maintain balance stabilization, and controller is designed by considering ZMP $(p)$ as the input to stabilize inverted pendulum. From Eqn. (2), considering the following dynamics,

$$
\ddot{l}=\theta-\frac{g}{l} p,
$$

and desired $\mathrm{ZMP}\left(p_{d}\right)$ which stabilizes inverted

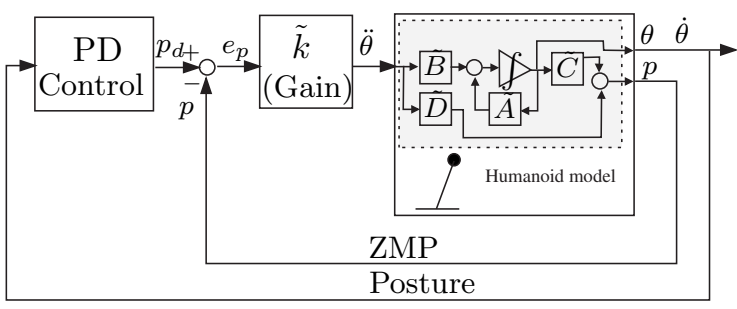

Fig. 2. Block diagram of inverted pendulum control and ZMP feedback control of humanoid robot

pendulum can be set using state feedback as follows,

$$
p_{d}=-\tilde{f}_{1} \theta-\tilde{f}_{2} \dot{\theta}
$$

where, $\tilde{f}_{1}$ and $\tilde{f}_{2}$ are state feedback gains. And then, balance stabilization is realized by tracking actual ZMP of robot to desired ZMP, and it is conducted by ZMP compensation control. 2.2 ZMP compensation control ZMP compensation control is used to track desired ZMP $\left(p_{d}\right)$ set by inverted pendulum control. ZMP compensation control is conducted by considering postural acceleration as the input, and difference between actual and desired ZMP is fedback as,

$$
\ddot{\theta}=-\tilde{k}\left(p-p_{d}\right),
$$

where, $\tilde{k}$ is output feedback gain and high gain feedback is mostly used. ZMP $(p)$ is as described in Eqn. (2).

2.3 Combination of inverted pendulum control and ZMP compensation control

Using inverted pendulum control and ZMP compensation control, define $\tilde{\xi}$ as new variable which describes difference between actual and desired $\mathrm{ZMP}$,

$$
\tilde{\xi}:=p-p_{d}=\left(l+\tilde{f}_{1}\right) \theta+\tilde{f}_{2} \dot{\theta}-\frac{l^{2}}{g} \ddot{\theta} .
$$

And then considering postural acceleration $\ddot{\theta}$ as input to system, entire system can be formulated in unified form as follows,

$$
\left(\begin{array}{c|c}
\tilde{\boldsymbol{A}} & \tilde{\boldsymbol{B}} \\
\hline \tilde{\boldsymbol{C}} & \tilde{\boldsymbol{D}}
\end{array}\right)\left\{\begin{aligned}
\frac{d}{d t}\left[\begin{array}{c}
\theta \\
\dot{\theta}
\end{array}\right] & =\left[\begin{array}{ll}
0 & 1 \\
0 & 0
\end{array}\right]\left[\begin{array}{c}
\theta \\
\dot{\theta}
\end{array}\right]+\left[\begin{array}{l}
0 \\
1
\end{array}\right] \ddot{\theta}, \\
y & =\left[l+\tilde{f}_{1} \tilde{f}_{2}\right]\left[\begin{array}{c}
\theta \\
\dot{\theta}
\end{array}\right]+\left[-l^{2} / g\right] \ddot{\theta}, \\
\ddot{\theta} & =-\tilde{k} y,
\end{aligned}\right.
$$

where, $y:=\tilde{\xi}$ is output of the system and input of the system $(\theta)$ is obtained by output feedback. Block diagram of this formulation can be shown in Fig.2, and there is feedthrough term from input $\ddot{\theta}$ to ZMP (output $(y)$ ) which makes the closed loop system difficult to analyze. That is why, state feedback gain $\tilde{f}_{1}, \tilde{f}_{2}$ and output feedback gain $\tilde{k}$ are difficult to find analytically and in most of cases it is required to find these feedback gains experimentally.

\section{HUMANOID ROBOT MODEL WITH COMPLIANCES}

Humanoid robot model considered in this paper is shown in Fig.3 and has compliances in sole 


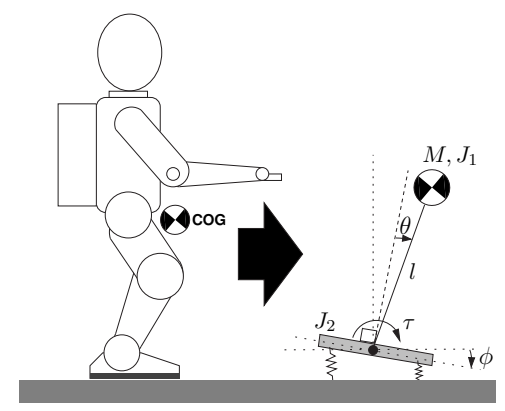

Fig. 3. COM model of humanoid robot with compliances

of the foot. Humanoid robot composed by many links can be described using dynamics of center of mass $(\mathrm{COM})$ and can be represented using 1 link inverted pendulum model. The compliances are modelled using linear spring. In this model, robot can move in $\theta$ and $\phi$ direction. It is assummed that moment inertia of robot is $J_{1}$ and total mass is $M$ and concentrated at position $l$ from ankle. Compliances are composed by 2 springs whose coefficients are $K_{1}$ and $K_{2}$ respectively, and are placed at position $r_{1}$ and $r_{2}$ from center of the foot. Reaction force by compliances can be calculated as $\left(K_{1} r_{1}^{2}+K_{2} r_{2}^{2}\right) \phi$ and to simplify the formulation, define $K:=\left(K_{1} r_{1}^{2}+K_{2} r_{2}^{2}\right)$. Gray part in Fig. 3 is foot and has moment inertia $J_{2}$ but the mass is neglected. Displacement of $\phi$ can not be measured, but it is assummed that force sensor is set up in sole of foot, so that ground reaction torque can be measured.

Equation motion of humanoid model mentioned above can be described as,

$$
\begin{aligned}
J_{1}(\ddot{\theta}+\ddot{\phi}) & =\tau+M g l \sin (\theta+\phi), \\
J_{2} \ddot{\phi} & =-\tau-K \phi .
\end{aligned}
$$

Eqn.(9) expresses the dynamics of COM, $\tau$ and $g$ are equivalent torque to move COM and gravitational acceleration respectively. Eqn. (10) expresses the dynamics of foot.

Using force sensor, ground reaction torque can be measured, however it does not describe ground reaction torque of $\mathrm{COM}$ but describes reaction torque from compliances in sole of foot. Thus, ground reaction torque can be described as,

$$
\bar{\tau}=-K \phi .
$$

The ground reaction torque $(\bar{\tau})$ and measured ZMP $\bar{p}$ have proportional relation and based on linearization of the model, this relation can be described as follow which shows that ground reaction force $\bar{\tau}$ and ZMP are equivalent.

$$
\bar{p}=-\frac{1}{M g} \bar{\tau} .
$$

Using Eqn.(10) and Eqn. (11), and using postural acceleration as the input, equation motion of ground reaction torque can be described as,

$$
\ddot{\bar{\tau}}=-\frac{K M g l}{J_{1}+J_{2}} \theta-\frac{M g l-K}{J_{1}+J_{2}} \bar{\tau}+\frac{K J_{1}}{J_{1}+J_{2}} \ddot{\theta} .
$$

Define postural angle and velocity, ground reaction torque and its derivative as states of the system, the dynamics of entire system can be described in state space form as,

$(\boldsymbol{A}, \boldsymbol{B})\left\{\frac{d}{d t}\left[\begin{array}{c}\theta \\ \dot{\theta} \\ \bar{\tau} \\ \dot{\bar{\tau}}\end{array}\right]=\left[\begin{array}{cccc}0 & 1 & 0 & 0 \\ 0 & 0 & 0 & 0 \\ 0 & 0 & 0 & 1 \\ a_{1} & 0 & a_{2} & 0\end{array}\right]\left[\begin{array}{c}\theta \\ \dot{\theta} \\ \bar{\tau} \\ \dot{\bar{\tau}}\end{array}\right]+\left[\begin{array}{c}0 \\ 1 \\ 0 \\ b_{1}\end{array}\right] \ddot{\theta}\right.$,

where, $a_{1}:=-\frac{K M g l}{J_{1}+J_{2}}, a_{2}:=-\frac{M g l-K}{J_{1}+J_{2}}$ and $b_{1}:=$ $\frac{K J_{1}}{J_{1}+J_{2}}$.

\section{FORMULATION OF ZMP CONTROL PROBLEM}

In this section, formulation of ZMP control problem of humanoid robot with compliances is described based on description in section 2. However, besides using ZMP $(p)$ treated in section 2 , ground reaction torque $(\bar{\tau})$ treated in section 3 is used instead. This ground reaction torque $(\bar{\tau})$ is measured using force sensor, and in the following description $\bar{\tau}$ is called ZMP.

As described in section 2, desired ZMP $\left(\bar{\tau}_{d}\right)$ can be set as a function of COM states, using state feedback gain $f_{1}, f_{2}$ as parameters, desired ZMP $\left(\bar{\tau}_{d}\right)$ can be set as,

$$
\bar{\tau}_{d}=-f_{1} \theta-f_{2} \dot{\theta}
$$

And then balance stabilization is maintained by tracking real ZMP to desired ZMP, and it is conducted using ZMP compensation control as described in section 2 using high gain feedback by considering postural acceleration as the input as follows,

$$
\ddot{\theta}=-k\left(\bar{\tau}-\bar{\tau}_{d}\right) .
$$

Define new variable $\xi$ as difference of actual and desired ZMP,

$$
\xi:=\bar{\tau}+f_{1} \theta+f_{2} \dot{\theta} .
$$

Tracking condition of actual ZMP to desired ZMP $\left(\bar{\tau} \rightarrow \bar{\tau}_{d}\right)$ can also be described as $\xi \rightarrow 0$ at $t \rightarrow \infty$.

Thus, balance stabilization of humanoid robot based on inverted pendulum control and ZMP compensation control can be described as output zeroing control of the following system using high gain feedback,

$$
\left(\begin{array}{r|r}
\boldsymbol{A} & \boldsymbol{B} \\
\hline \boldsymbol{C} & \mathbf{0}
\end{array}\right)\left\{\begin{aligned}
\frac{d \boldsymbol{x}}{d t} & =\boldsymbol{A} \boldsymbol{x}+\boldsymbol{B} u \\
y & =\left[\begin{array}{llll}
f_{1} & f_{2} & 1 & 0
\end{array}\right] \boldsymbol{x} \\
u & =-k y
\end{aligned}\right.
$$

where, $\boldsymbol{x}:=[\theta, \dot{\theta}, \bar{\tau}, \dot{\bar{\tau}}]^{T}, u:=\ddot{\theta}, y:=\xi$.

Output feedback gain $k$ corresponds to high gain feedback to make output $y=0$. Inverted pendulum control of the system to generate desired ZMP has characteristic that feedback of $\dot{\bar{\tau}}$ is not used. Zeroing the output of the system will make actual ZMP tracks desired ZMP, however the system described in Eqn. (18) is 4th order system, and relative degree of output function is 1 . Thus when 
actual ZMP completely tracks desired ZMP, there will be 3 dimensional zero dynamics occurred as internal dynamics.

Balance stabilization based on ZMP principle is conducted by tracking actual ZMP to desired $\mathrm{ZMP}$, as a consequence ground reaction force in the sole is controlled, and from Eqn.(11), it means that displacement of foot $\phi$ is controlled.

By considering compliances in humanoid robot model, feedthrough term from postural acceleration $(\ddot{\theta})$ to $\mathrm{ZMP}(p)$ does not appear in the formulation of ZMP control problem. Thus, balance stability analysis is easy to handle from control point of view.

\section{STABILITY OF ZERO DYNAMICS}

Since relative degree of output function in the system formulated in previous section is 1 , when output of system completely $y=0$, there will be 3 dimensional zero dynamics in the system. Then the behavior of system is decided by this zero dynamics, thus it is needed to consider the stability of zero dynamics. In linear system, zero dynamics corresponds to zeros of the system, so there will be poles of the system which converges to point at infinity.

Since there are free parameters $f_{1}$ and $f_{2}$ in the system, zero dynamics will vary depends on these free parameters. Thus, it is needed to use appropriate state feedback gain. The zero dynamics of the system can be described as,

$$
\begin{aligned}
& f_{2}\left(J_{1}+J_{2}\right) s^{3}+\left(f_{1}\left(J_{1}+J_{2}\right)+J_{1} K\right) s^{2}+ \\
& f_{2}(K-M g l) s+f_{1}(K-M g l)-M g l K=0 .
\end{aligned}
$$

Using Routh-Hurwitz stability criterion, necessary and sufficient condition of stability of zero dynamics can be described as,

$$
\begin{aligned}
& f_{1}>\frac{M g l K}{K-M g l}, \\
& f_{2}>0 .
\end{aligned}
$$

However because of nonlinearity and friction, balance stabilization can not be maintained only by choosing high gain feedback which fulfills above condition. Furthermore, it is difficult to find feedback gains which conduct good performance in stabilizing inverted pendulum. Thus, it is needed to find appropriate state feedback gain $f_{1}, f_{2}$ and output feedback gain $k$, and in this paper the guideline of choosing these parameters is proposed by focusing on the following items.

- The behavior of zero dynamics is considered based on compliances change $K$, and then guidelines to decide state feedback gain $f_{1}$ and $f_{2}$ are provided (section 6 ).

- The behavior of closed loop system is considered based on output feedback gain $k$, and then guidelines to decide output feedback gain $k$ is provided (section 7 ).
Table 1. Robot parameters in analysis

\begin{tabular}{|l|cl|}
\hline Total mass $(M)$ & 50.0 & {$[\mathrm{~kg}]$} \\
\hline gravitational acceleration $(g)$ & 9.8 & {$\left[\mathrm{~m} / \mathrm{s}^{2}\right]$} \\
\hline Length of $\operatorname{link}(l)$ & 1.0 & {$[\mathrm{~m}]$} \\
\hline Moment inertia of $\operatorname{link}\left(J_{1}\right)$ & 50.0 & {$\left[\mathrm{~kg} \cdot \mathrm{m}^{2}\right]$} \\
\hline Moment inertia of $\operatorname{foot}\left(J_{2}\right)$ & 0.3 & {$\left[\mathrm{~kg} \cdot \mathrm{m}^{2}\right]$} \\
\hline
\end{tabular}

To conduct root locus analysis, humanoid robot parameters described in Table 1 are used.

6. ANALYSIS OF ZERO DYNAMICS CONSIDERING COMPLIANCES CHANGE

Zero dynamics from Eqn.(19) can be rewritten in the following form,

$$
\begin{array}{r}
f_{2}\left(J_{1}+J_{2}\right) s^{3}+f_{1}\left(J_{1}+J_{2}\right) s^{2}-f_{2} M g l s-f_{1} M g l+ \\
K\left(J_{1} s^{2}+f_{2} s+f_{1}-M g l\right)=0 .
\end{array}
$$

When $K \rightarrow 0$, the dynamics $D(s):=f_{2}\left(J_{1}+\right.$ $\left.J_{2}\right) s^{3}+f_{1}\left(J_{1}+J_{2}\right) s^{2}-f_{2} M g l s-f_{1} M g l=0$ becomes dominance, and this part is called compliance mode. Thus, compliance mode corresponds to humanoid robot which has very flexible compliances in sole. In this mode, the poles of zero dynamics can be described as,

$$
p_{1,2,3}=-\frac{f_{1}}{f_{2}}, \pm \sqrt{\frac{M g l}{J_{1}+J_{2}}} .
$$

On the other hand, when $K \rightarrow \infty$, the dynamics $N(s):=J_{1} s^{2}+f_{2} s+f_{1}-M g l=0$ becomes dominance, and this part is called rigid mode (inverted pendulum mode). Thus, inverted pendulum mode corresponds to humanoid robot which has rigid foot. This mode is equivalent to inverted pendulum mode in Nagasaka (Nagasaka et al. (1999)), and the poles of zero dynamics can be described as,

$$
z_{1,2}=\frac{-f_{2} \pm \sqrt{f_{2}^{2}-4 J_{1}\left(f_{1}-M g l\right)}}{2 J_{1}} .
$$

Since root locus of Eqn.(21) and $N(s) / D(s)$ coincides, the behavior of root locus of $N(s) / D(s)$ is considered, and then the root locus is classified depend on the shapes with state feedback gain $f_{1}$ and $f_{2}$. This classification is described below.

First, from necessary and sufficient condition that zero dynamics become stable, the boundary of stable area and unstable area can be described as,

$$
a: f_{1}=\frac{M g l K}{K-M g l}
$$

$f_{1}>(M g l K) /(K-M g l)$ represents stable area, while $f_{1}<(M g l K) /(K-M g l)$ represents unstable area.

Then, the poles of inverted pendulum mode $z_{1}, z_{2}$ can have complex or real number, from Eqn.(23) the boundary of complex and real area can be described as,

$$
b: f_{1}=\frac{1}{4 J_{1}} f_{2}^{2}+M g l
$$

$f_{1}>f_{2}^{2} /\left(4 J_{1}\right)+M g l$ represents complex area, while $f_{1}<f_{2}^{2} /\left(4 J_{1}\right)+M g l$ represents real area. 

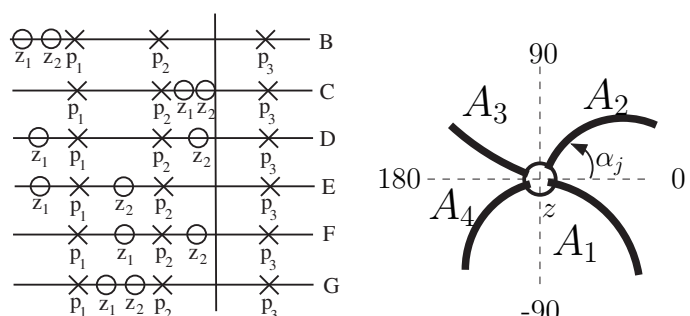

$-90$

(a) Positional relationship (b) Convergence angle to between $z_{1}, z_{2}, p_{1}, p_{2}$ and inverted pendulum model $p_{3}$

Fig. 4. Positional relationship of poles \& zeros and convergence angle

Then, if $z_{1}, z_{2}$ are real, then feasible positonal relationship between $z_{1}, z_{2} p_{1}, p_{2}$ and $p_{3}$ can be shown in Fig.4(a). Using Eqn.(22) and Eqn.(23) the boundary of this positional relationship can be described as,

$$
\begin{aligned}
& c: f_{1}=\sqrt{\frac{M g l}{J_{1}+J_{2}}} f_{2} \\
& d: f_{1}=\sqrt{\frac{M g l}{J_{1}}} f_{2} \\
& e: f_{1}=\sqrt{\frac{M g l}{J_{1}+J_{2}}} f_{2}+M g l-\frac{M g l J_{1}}{J_{1}+J_{2}}
\end{aligned}
$$

Furthermore if $z_{1}, z_{2}$ are complex number, shape of root locus changes depend on convergence angle of zero dynamics to poles of inverted pendulum $z_{1}$, $z_{2}$. This convergence angle can be found using the following formula (T. Sugie (2001)),

$$
\alpha_{j}=180^{\circ}+\sum_{i=1}^{3} \angle\left(z_{j}-p_{i}\right)-\sum_{i \neq j} \angle\left(z_{j}-z_{i}\right),
$$

where, $p_{i}, z_{j}$ are as described in Eqn.(22) and Eqn.(23). From this results, complex area of poles of inverted pendulum mode $z_{1}, z_{2}$ can be partitioned into 4 areas depend on convergence angle shown in Fig.4(b). Each boundary of area is difficult to describe as a function of feedback gain $f_{1}$ and $f_{2}$, in this paper this area is found numerically.

Stability of zero dynamics is analyzed by varying the value of $K$ and focusing on the motion of root locus of poles of zero dynamics in each area. The analysis which is conducted here, corresponds to changing the value of $K \rightarrow \infty$ to $K=0$. The result is explained below, and to distinct the plot, poles of inverted pendulum model is represented by mark $\bigcirc$ and poles of feedback mode is represented by mark $x$.

- Area $A_{1}$ : In this area, poles of inverted pendulum mode are complex, and convergence angle is $-90^{\circ}<\alpha<0^{\circ}$. By changing the value of $K$, root locus is shown in Fig.5(a).

- Area $A_{2}$ : In this area, poles of inverted pendulum mode are complex, and convergence angle is $0^{\circ}<\alpha<90^{\circ}$. By changing the value of $K$, root locus is shown in Fig.5(b).

- Area $A_{3}$ : In this area, poles of inverted pendulum mode are complex, and convergence
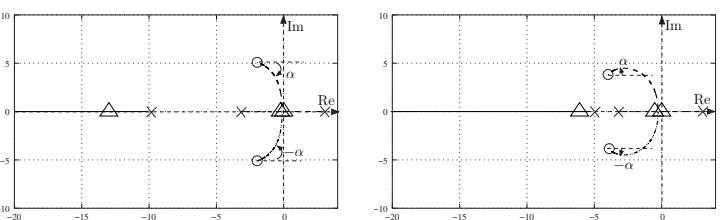

(a) $A_{1}\left(f_{1}=2000, f_{2}=200\right)$

(b) $A_{2}\left(f_{1}=2000, f_{2}=400\right)$
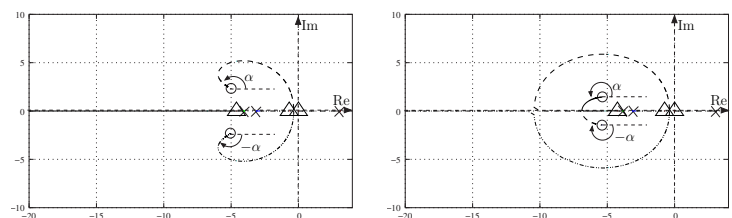

(c) $A_{3}\left(f_{1}=2000, f_{2}=500\right)$

(d) $A_{4}\left(f_{1}=2000, f_{2}=530\right)$
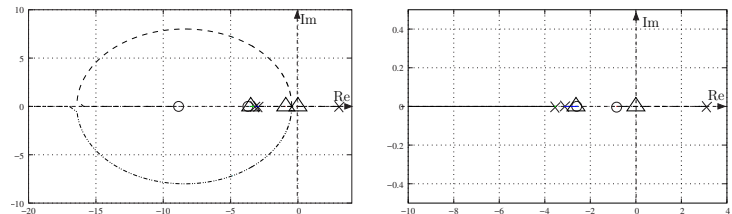

(e) $B\left(f_{1}=2000, f_{2}=600\right)$
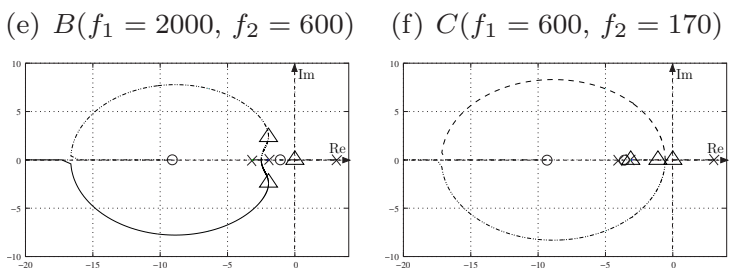

(g) $D\left(f_{1}=1000, f_{2}=500\right)$

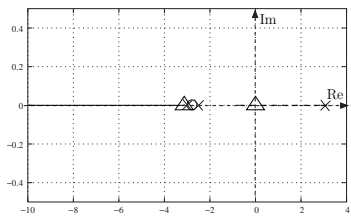

(h) $E\left(f_{1}=1877, f_{2}=600\right)$

(i) $F\left(f_{1}=626.5, f_{2}=200\right)$

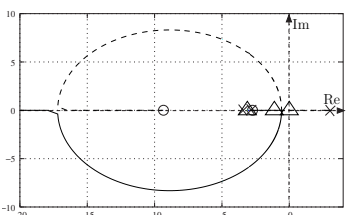

(j) $G\left(f_{1}=1874, f_{2}=600\right)$

Fig. 5. Root locus of zero dynamics

angle is $90^{\circ}<\alpha<180^{\circ}$. By changing the value of $K$, root locus is shown in Fig.5(c).

- Area $A_{4}$ : In this area, poles of inverted pendulum mode are complex, and convergence angle is $180^{\circ}<\alpha<270^{\circ}$. By changing the value of $K$, root locus is shown in Fig.5(d).

- Area $B:$ In this area, poles of inverted pendulum mode are real, and convergence angle is $0^{\circ}$ and $180^{\circ}$. By changing the value of $K$, root locus is shown in Fig.5(e).

- Area $C$ : In this area, poles of inverted pendulum mode are real, and convergence angle is $0^{\circ}$ and $180^{\circ}$. By changing the value of $K$, root locus is shown in Fig.5(f).

- Area $D:$ In this area, poles of inverted pendulum mode are real, and convergence angle is $0^{\circ}$ and $180^{\circ}$. By changing the value of $K$, root locus is shown in Fig.5(g).

- Area $E:$ In this area, poles of inverted pendulum mode are real, and convergence angle is $180^{\circ}$ and $180^{\circ}$. By changing the value of $K$, root locus is shown in Fig.5(h).

- Area $F$ : In this area, poles of inverted pendulum mode are real, and convergence angle 


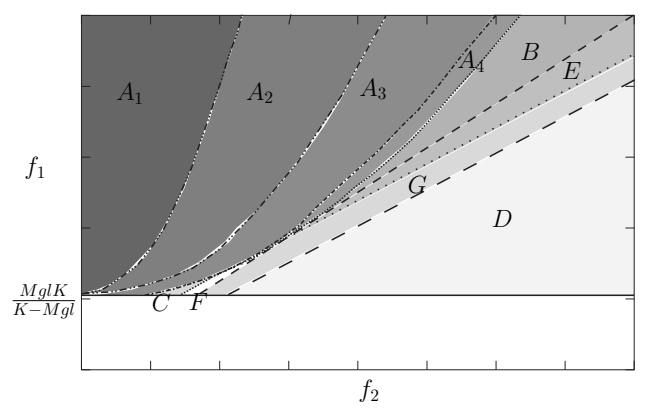

Fig. 6. Classification of root locus by shapes

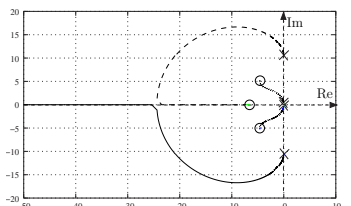

(a) $f_{1}=2000, f_{2}=500$

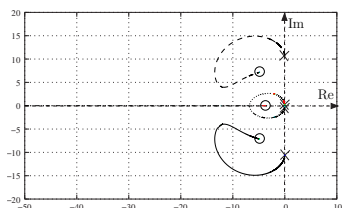

(b) $f_{1}=2000, f_{2}=600$
Fig. 7. Root locus of closed loop system

is $0^{\circ}$ and $0^{\circ}$. By changing the value of $K$, root locus is shown in Fig.5(i).

- Area $G$ : In this area, poles of inverted pendulum mode are real, and convergence angle is $0^{\circ}$ and $180^{\circ}$. By changing the value of $K$, root locus is shown in Fig. $5(\mathrm{j})$.

Eventually the shapes of root locus of zero dynamics can be classified into 10 areas as shown in Fig.6.

From analysis results of root locus of zero dynamics, to realize balance stabilization based on ZMP principle, area $A_{4}, B, D$ and $F$ can be considered to have relative good performance. In these areas, degradation from compliances effect can be suppressed. And since poles of the system which converge to point at infinity only 1 , it is always move to stable direction.

From graphs of root locus, there is one unstable pole in inverted pendulum mode, and the boundary value of this pole can be found using RouthHurwitz stability criterion, and if the value of compliance $K$ fulfills the following condition, then system becomes unstable.

$$
K<\frac{f_{1} M g l}{f_{1}-M g l}
$$

This boundary condition is shown in the graphs by mark $\triangle$.

\section{ANALYSIS OF ZERO DYNAMICS CONSIDERING OUTPUT FEEDBACK}

To maintain balance stabilization, it is needed to conduct output zeroing control of the system described in Eqn.(18). It is realized using output feedback corresponds to minor feedback, and it is known that balance stabilization can be maintained using output feedback only (Nagasaka et al. (1999)).

Using $u:=-k y=-k \boldsymbol{C} \boldsymbol{x}$ as the input, then characteristics equation of closed loop system can be described as,

$$
\begin{gathered}
s^{4}+\frac{K-M g l}{J_{1}+J_{2}} s^{2}+\frac{k}{J_{1}+J_{2}}\left(f_{2}\left(J_{1}+J_{2}\right) s^{3}+\right. \\
\left(f_{1}\left(J_{1}+J_{2}\right)+J_{1} K\right) s^{2}+f_{2}(K-M g l) s+ \\
\left.f_{1}(K-M g l)-M g l K\right)=0 .
\end{gathered}
$$

When $k \rightarrow \infty$, the dynamics $N(s):=f_{2}\left(J_{1}+\right.$ $\left.J_{2}\right) s^{3}+\left(f_{1}\left(J_{1}+J_{2}\right)+J_{1} K\right) s^{2}+f_{2}(K-M g l) s+$ $f_{1}(K-M g l)-M g l K=0$ becomes dominance, and this dynamics describes zero dynamics analyzed in section 6 .

On the other hand, when $k \rightarrow 0$, the dynamics $D(s):=s^{4}+(K-M g l) /\left(J_{1}+J_{2}\right) s^{2}=0$ becomes dominance, and the poles of control system can be found as,

$$
s_{1,2,3,4}=0,0, \pm \sqrt{\frac{K-M g l}{J_{1}+J_{2}}} i .
$$

Consequently, considering behavior of root locus of $N(s) / D(s)$, output feedback gain $k$ is varied. Root locus of control system when 2 types of state feedback gain are selected, is shown in Fig.7(a) and Fig.7(b). Here, poles of zero dynamics is represented by mark $\bigcirc$, and poles of control system when $k \rightarrow 0$ is represented by mark $\times$.

From these results, it is needed to used output feedback gain $k$ as big as possible. In this case, control system becomes stable and degradation of system response by compliances can be minimized.

\section{CONCLUSIONS}

This paper analyzed balance stabilization of humanoid robot based on ZMP principle. Balance stabilization problem based on ZMP principle is formulated by inverted pendulum control and ZMP compensation control, and can be described as output zeroing control and the existence of zero dynamics. The condition to stabilize total system is derived and the guidelines to choose controller parameters are proposed based on root locus analysis of zero dynamics and control system.

\section{REFERENCES}

K. Hirai, M. Hirose, Y. Haikawa, and T. Takenaka. The development of honda humanoid robot. In Proceedings of the 1998 IEEE International Conference on Robotics \& Automation, 1998.

A. Isidori. Nonlinear control systems. Springer Verlag, 1995.

K. Nagasaka, M. Inaba, and H. Inoue. Stabilization of dynamic walk on a humanoid using torso position compliance control. In Proceedings of the 19th Annual Conference of the Robotics Society of Japan, pages 1193-1194 (in Japanese), 1999.

M. Fujita T. Sugie. Introduction to Feedback Control. Corona (in Japanese), 2001.

M. Vukobratovic, B. Borovac, D. Surla, and D. Stokic. Biped Locomotion: Dynamics, Stability, Control and Application. Springer Verlag, 1975 . 